Accepted for publication in "Cognitive Behaviour Therapy"

Note: This is an uncorrected version of an author's manuscript accepted for publication. Copyediting, typesetting, and review of the resulting proofs will be undertaken on this manuscript before final publication. During production and pre-press, errors may be discovered that could affect the content.

\title{
Positive Memory Enhancement Training for Individuals with Major Depressive
}

Disorder

Kimberly A. Arditte Hall ${ }^{1} \quad$ Rudi De Raedt ${ }^{2} \quad$ Kiara R. Timpano $^{3}$ Jutta Joormann ${ }^{4}$

${ }^{1}$ National Center for Posttraumatic Stress Disorder, VA Boston Healthcare System, 150 S.

Huntington Ave. (116B), Boston, MA, 02130

2 Department of Experimental Clinical and Health Psychology, Ghent University, Henri

Dunantlaan 2, B-9000, Ghent, Belgium

3 Department of Psychology, University of Miami, 5665 Ponce de Leon Blvd., Coral Gables,

FL, 33146

4 Department of Psychology, Yale University, PO Box 208205, New Haven, CT, 06511

Author Note: The views expressed in this article are those of the authors and do not necessarily represent the views of the Department of Veterans Affairs or the United States Government.

Disclosure Statement: Dr. De Raedt received a Travel Grant from the Research Foundation Flanders (V4.040.10N). Dr. Arditte Hall declares that she has no conflict of interest. Dr. Timpano declares that she has no conflict of interest. Dr. Joormann declares that she has no conflict of interest. 


\begin{abstract}
When in a negative mood state, individuals with major depressive disorder (MDD) may have difficulties recalling positive autobiographical memories in a manner that repairs that negative mood. Using cognitive bias modification techniques investigators have successfully altered different aspects of cognition among individuals with MDD. However, little has been done to investigate the modification of positive autobiographical memory recall. This study examined the impact of a novel positive memory enhancement training (PMET) on the memories and subjective affective experiences of individuals with MDD $(N=$ 27). Across a series of training trials, participants first recalled a specific sad memory in order to elicit a negative mood state. They then recalled a happy memory and completed procedures to elicit a vivid, here-and-now quality of the memory. PMET procedures were hypothesized to promote mood repair via the recall of increasingly vivid and specific positive memories. Over the course of training, PMET participants demonstrated improved memory specificity and greater perceived ability to "relive" positive memories. The procedures were also found to repair mood; PMET participants' affect following recall of positive memories did not differ from control participants' affect following recall of neutral memories. Results provide preliminary support for PMET as a method to improve the quality of positive memories and facilitate emotion regulation among individuals with MDD.
\end{abstract}

Keywords: Depression; Memory Bias; Cognitive Bias Modification; Emotion Regulation; Mood Repair 


\section{Positive Memory Enhancement Training for \\ Individuals with Major Depressive Disorder}

Supported by more than 50 years of theory and research, the role of cognition in the onset and maintenance of major depressive disorder (MDD) is well established (Beck, 1964; Beck, 2005). Individuals with MDD often engage in maladaptive cognitive styles, endorse depressive schemas, and display deficits in cognitive control (see Joormann \& Arditte, 2014, for a review). Furthermore, depression is linked with particular patterns of information processing (e.g., cognitive biases), which may set the stage for emotion regulation difficulties (Joormann \& D’Avanzato, 2010). Depression-related biases have been documented within cognitive domains, such as attention, interpretation, and memory (Everaert, Koster, \& Derakshan, 2012; Lemogne et al., 2006). For example, when compared with their non-depressed counterparts, individuals with MDD may experience facilitated recall of negative memories and, conversely, impaired recall of positive memories (Claúdio, Aurélio, \& Machado, 2012). Results of this nature have led investigators to modify cognitive biases using experimental paradigms (Hallion \& Ruscio, 2007). However, few studies have examined the modification of memory biases in individuals with MDD.

Research investigating memory and depression has resulted in consistent support for two main findings. First, individuals with MDD often demonstrate emotion-specific patterns of memory recall. Depression has been associated with more frequent intrusive thoughts about negative past events (Brewin, Gregory, Lipton, \& Burgess, 2010). Within the laboratory, depressed participants have shown a bias towards recalling negative rather than positive memories, and have recalled a greater number of negative memories than either persons with panic disorder or non-disordered controls (Claúdio et al., 2012). 
Conversely, individuals with MDD demonstrate difficulty recalling positive memories. Results link depression with the recall of fewer positive memories and slower recall following positive prompts (Gupta \& Kar, 2012; Young, Erickson, \& Drevets, 2012).

Research has also revealed that depressed persons recall generic autobiographical experiences, even when prompted to recall specific past events (i.e., overgeneral memory [OGM]; Williams et al., 2007; Sumner, Griffith, \& Mineka, 2010). Rather than recalling an event that happened on a particular day and time, depressed persons are more likely to recall a general class of events or an event occurring over an extended period of time. OGM has been associated with a ruminative cognitive style and difficulties with problem solving, as well as with delayed recovery from major depressive episodes (Peeters, Wessel, Merckelbach, \& Boon-Vermeeren, 2002; Raes et al., 2005; Sumner et al., 2010). Notably, studies also show that OGM is amenable to change (Neshat-Doost et al., 2013), making this construct a potentially efficacious target for intervention.

Though results are mixed (e.g., Sumner et al., 2010), some studies indicate that OGM is particularly pronounced when depressed persons are cued to recall positive memories (Gupta \& Kar, 2012; Young et al., 2012). Compared to healthy controls, depressed persons are less likely to recall specific positive memories when cued and are more likely to use an observer (i.e., observing a scene unfold from a third person perspective), rather than a field perspective (i.e., observing a scene unfold from a first person perspective; Lemogne et al., 2006). Because an observer perspective is often associated with emotional distancing, use of a field perspective could allow for greater engagement with positive emotions.

The manner in which depressed persons recall positive autobiographical memories may have important implications for their ability to regulate acute negative emotions. 
Researchers have found that, among non-depressed individuals, positive memories can be used to effectively repair acute sadness; in contrast, the recall of positive memories appears to have no impact on sad mood among individuals with subclinical or remitted depression, and may even contribute to an increase in sadness among currently depressed individuals (Joormann \& Siemer, 2004; Joormann, Siemer, \& Gotlib, 2007). The reasons why depressed persons have difficulty utilizing positive memories to repair sad mood remain unclear, but characteristics of their positive memories (e.g., use of an observer perspective, lack of vivid and specific details) could play an important role. Enhancing such characteristics may improve depressed individuals mood by allowing them to reexperience the memory, and engage with its associated affect, more fully. In support of this hypothesis, results from an unselected sample indicated that training participants to utilize a field perspective while recalling positive memories contributed to greater subjective positive affect (Holmes, Coughtrey, \& Conner, 2008).

Cognitive bias modification (CBM) is an approach to reducing clinical symptoms by targeting relevant cognitive biases. Because cognitive biases are habitual and often occur outside of conscious awareness, CBM procedures seek to modify biases through repeated experiential practice (see Beard, 2011 for a more thorough discussion). To date, few studies have used CBM to enhance positive memory recall in depression, and studies that have (e.g., Watkins, Baeyens, \& Read, 2009) targeted OGM more generally. Conceptualizing positive memory recall as a strategy that can be used to repair negative moods, depressed persons may benefit from targeted CBM techniques that promote the vivid, experiential recall of positive memories in the context of an acute negative mood state. To date, no study has directly targeted depressed persons' positive memories in this way. 
This study examined the use of a positive memory enhancement training (PMET) for individuals with MDD. Training procedures were developed to augment the quality of positive memories as a method for repairing acute sadness, and were compared to a control condition in which participants were trained to recall neutral memories. The impact of training on the quality of recalled memories was examined across study procedures. The first (1) aim of the study was to examine the impact of the procedures to promote the recall of vivid and specific memories. Because both conditions completed these procedures, participants in both the PMET and control conditions were expected to demonstrate improvement in this area (1a) and were not expected to differ from each other (1b). The second (2) aim of the study was to examine the impact of recalling a positive memory on PMET participants' ability to repair an induced sad mood. We hypothesized that PMET participants would demonstrate initial difficulty repairing sad mood through the recall of positive memories (i.e., PMET participants would continue to endorse more sadness and less happiness than control participants; 2a). However, we also hypothesized that PMET participants' ability to repair their sad mood would increase over the course of training (i.e., PMET and control participants would not differ in endorsed sadness and happiness ratings following positive memory recall; $2 \mathrm{~b}$ ). The third (3) aim of the study was to examine the impact of PMET on participants' ability to "relive" positive memories. PMET participants were expected to demonstrate an increase in perceived ability to relive positive memories over the course of the training (3a). We also hypothesized that the intensity of positive affect following positive memory recall would be correlated with the degree of perceived ability to relive the memory among PMET participants (3b). 


\section{Method}

\section{Participants}

Individuals from the community were invited to participate in a brief phone interview to assess for initial inclusion/exclusion criteria. Participants were required to be 18 to 60 years old and fluent in English, and were excluded if they met DSM-IV-TR criteria for a lifetime history of mania or psychotic symptoms, current alcohol or substance abuse, or a history of traumatic brain injury. Eligible individuals were invited to the laboratory to participate in a more comprehensive clinical interview.

During this session, participants completed the Structured Clinical Interview for the DSM-IV (SCID; First, Spitzer, Gibbon, \& Williams, 2002) with a trained and supervised graduate-level clinician. The SCID is a semi-structured diagnostic interview, which assesses for current and lifetime DSM-IV-TR Axis I disorders, that demonstrates good diagnostic reliability (Shear et al., 2000). Individuals were eligible to participate in subsequent study procedures if they met criteria for current MDD (interrater reliability of MDD diagnoses was 100\%). Comorbid diagnoses included social anxiety disorder (48.3\%), specific phobia (44.8\%), posttraumatic stress disorder (24.1\%), generalized anxiety disorder (20.7\%), panic disorder (17.2\%), and hoarding disorder (6.9\%). At the completion of the session, participants were assigned to either the PMET or the control condition using a counterbalanced design (i.e., condition assignment alternated between PMET and control).

A sample of 29 participants returned to complete a second study session, consisting of the study procedures described below. Procedures were prematurely terminated for two participants who were unable to generate autobiographical memories in response to the cues provided. The individuals who terminated procedures prematurely did not appear to 
differ from other participants on demographic or depression-related characteristics of interest. However, both were assigned to the PMET condition.

The final sample $(N=27$; PMST $n=13$; $\operatorname{control} n=14)$ had a mean age of $40.59(S D$ $=12.65$ ) years and was $56 \%$ female. Thirty-three percent identified as White, $33 \%$ as Black/African American, 26\% as Hispanic, 4\% as Asian, and 4\% as "Other." Assessment of current treatment status revealed that one (4\%) individual endorsed current psychotherapy, whereas five (19\%) endorsed current use of psychotropic medications (in all cases an SSRI). Mean depression severity on the Beck Depression Inventory-II (Beck, Steer, \& Brown, 1996) fell within the "severe" range $(M=30.04, S D=13.43)$. In addition, the mean number of depressive episodes was $3.95(S D=4.63)$, though $6(22 \%)$ participants were unable to estimate the number of episodes they had experienced or stated that they had experienced too many episodes to count. Conditions did not differ with regard to any of the demographic variables, depression-related characteristics, or other comorbid conditions ( $p s>.10$ ), with the exception of generalized anxiety disorder, which was present at a higher rate in the PMET condition (38\%) as compared to the control condition (7\%), $\chi^{2}=6.74, d f=2, p=.03$.

\section{Positive Memory Enhancement Training (PMET)}

The study protocol was reviewed and approved by the (Blinded for Review)'s Institutional Review Board. Informed consent was obtained from all individuals included in the study. Participants were told that the purpose of the study was to investigate their ability to vividly imagine past events and that, to do this, they would be asked to recall memories out loud. They were not provided with explicit information about the study aims or hypotheses. Participants then completed training procedures, which comprised both a 
practice and a training phase. Sessions took approximately 2.5 hours to complete and participants were compensated $\$ 15 /$ hour for their time.

Practice Phase. During the practice phase, participants first practiced using mental imagery, by completing an exercise in which they were instructed to imagine holding a lemon. Participants were then provided with prompts (e.g., What do you see? Smell? Feel?) to allow them to experience the lemon in their mind's eye in a multi-sensory manner.

Following the lemon exercise, participants were provided with instructions on identifying and recalling autobiographical memories. Participants were told that they would be given a cue, representing a common event (e.g., going shopping). They were then asked to "remember a specific, personal memory" related to that cue "of an event that happened on a particular day, more than one week ago." To practice these procedures, participants were asked to recall two memories related to the cue, "trip or vacation." Prior to the recall of each memory, the experimenter provided an example to ensure that participants understood the instructions.

Within the PMET condition, participants first recalled a sad memory. Participants were given 3 minutes to recall their memory out loud, while the experimenter listened to ensure that participants recalled a sad memory and that the memory represented a specific event, occurring on a single day. Participants were asked to recall additional sad memories until both of these conditions were met. Next, participants were asked to recall a happy memory using the same cue. Participants were, again, given 3 minutes to recall the memory out loud, while the experimenter listened to ensure memories were both positive and specific. In addition, following the recall of this happy memory, participants completed procedures to assist them in using a field perspective. Participants were instructed to shut 
their eyes and "imagine in [their] mind, as vividly and concretely as possible, a 'movie' of how the event unfolded." They were, then, given 1 minute to recall the memory in the firstperson, present tense. Afterwards, the experimenter used prompts to enhance the quality of the memory (i.e., questions about what the participant could see, hear, and feel in their body as they "relived" the memory). Finally, participants were provided with 30 seconds to silently experience the memory.

Participants assigned to the control condition completed the same practice procedures as participants within the PMET condition with one exception. Rather than recalling one sad and one happy memory, control participants were asked to recall two neutral memories about events, in which the participant "experienced no strong negative or positive emotions." Following the second neutral memory, control participants completed the procedures to promote vivid and detailed recall of the memory.

Training Phase. In the training phase, participants were asked to recall a series of 16 memories related to 8 cues. The procedures for each cue took approximately 10 minutes to complete. As with the practice phase, participants in the PMET condition were first given 3 minutes to recall a sad memory for a particular cue and then given 3 minutes to recall a happy memory for that same cue. Happy memories were followed by the procedures to improve vivid and detailed recall described above. Within the control condition, participants were given two 3-minute periods to recall two neutral memories per cue. The second neutral memory was followed by procedures to improve vivid and detailed recall. Herein, the term Memory 1 is used to refer to the sad and corresponding neutral memories recalled by the PMET and control conditions, respectively, whereas the term Memory 2 
refers to the happy and corresponding neutral memories recalled by the PMET and control conditions, respectively.

Across all eight cues, subjective affect was assessed following the recall of Memory 1 and following the procedures to improve the vividness of Memory 2. At each time point, participants rated their current sadness and happiness using two Likert items, with anchors 1 (not at all) to 9 (extremely). At each of the Memory 2 time points, participants also rated their perceived ability to relive the memory by responding to the question, "to what extent were you able to experience this event through your own eyes, as if you were actively involved?" using a 5-point Likert scale with anchors 1 (not at all) to 5 (extremely).

\section{Data Preparation}

Coding Memory Data. Audio recordings of memories were collected from a subsample of $n=14$ participants (8 PMET and 6 control). Available recordings were transcribed and coded by an undergraduate research assistant, who was blind to the study hypotheses, and who had received extensive training in coding procedures. Memories were coded for valence and specificity. Valence was rated on a 7-point Likert scale with anchors, 1 (extremely negative) to 7 (extremely positive). In addition, specificity was examined by coding each memory as "generic" (i.e., representing a general class of events or an event occurring over an extended period of time) or "specific" (i.e., representing a single event occurring on a particular day). To assess reliability of ratings, $30 \%$ of the data was randomly selected for re-coding. Results revealed strong interrater reliability across valence ratings, ICC $(2,1)$, absolute $=.90$, and substantial reliability across specificity ratings, kappa $=.61, p<.001,95 \% \mathrm{CI}(.36, .86)$. 
Missing Data. Approximately 2\% of self-report data were missing, with at least some data missing from $n=6$ participants. Data appeared to be missing at random. To avoid reducing power to detect significant effects, multiple imputation (MI) was employed to handle missing values. MI is a well-regarded procedure in which missing values are replaced by multiple sets of simulated imputed values (Schafer \& Graham, 2002). Results are then combined to create a pooled estimate across imputed datasets. Data were imputed using fully conditional specification, an iterative Markov chain Monte Carlo (MCMC)

method. This MCMC method uses a participant's responses to all other variables of interest as predictors of each missing data value. The current study utilized procedures producing five imputations (Collins, Shafer, \& Kam, 2001).

\section{Data Analytic Plan}

Analyses were conducted using data from the beginning (Cue 1), middle (Cue 4), and end (Cue 8) of the training. The decision to do so was based upon both conceptual and methodological considerations. We expected effects would emerge over the completion of the training procedures for several cues, as opposed to on a cue by cue basis. In addition, providing data from 3 , rather than 8 , time points, reduced the number of comparisons that were calculated, and thus, the likelihood of reporting a Type I error. However, to ensure results were not unique to Cues 1,4 , and 8 , all 2x3 ANOVAs were also conducted as $2 \times 8$ ANOVAs. Except where otherwise noted, results were consistent across the $2 \times 3$ and $2 \times 8$ models.

To examine whether participants recalled sad, happy, or neutral autobiographical memories as instructed, coded valence ratings were examined using a 2x3 ANOVA, with condition (PMET vs. Control) as a between-subjects factor and time (Cue 1, Cue 4, and Cue 
8) as a within-subjects factor. A similar 2x3 ANOVA was used to examine memory specificity at Cues 1, 4, and 8, as a function of condition (Aim 1). Where appropriate, simple effects were examined using $t$-tests.

To examine the impact of the training on participants' subjective affective experiences, a series of 2x3 ANOVAs were used. First, participants' sadness and happiness ratings following the recall of Memory 1 at Cues 1, 4, and 8, were examined between participants in the PMET and control conditions. In order to examine whether PMET facilitated mood repair (Aim 2), difference scores were created to reflect the change in sadness and happiness from Memory 1 to Memory 2 at each cue. Difference scores were then analyzed between conditions and over time. Lastly, the effect of condition on sadness and happiness ratings following Memory 2 at Cues 1, 4, and 8 was examined. Again, where appropriate, follow up $t$-tests were conducted.

Finally, change in PMET participants' perceived ability to relive positive memories across the training was examined using a repeated-measures ANOVA and simple effects were followed up with paired samples $t$-tests (Aim 3a). Next, a series of Pearson's correlations were used to examine the associations between perceived ability to relive positive memories and happiness ratings at each time point (Aim 3b).

\section{Results}

\section{Examination of Recalled Memories}

Analysis of Memory 1 data revealed a main effect of condition, $F(1,10)=43.40, p<$ .001 , indicating that sad memories were rated by a coder as more negative than neutral memories $(d=2.06)$. Likewise, analysis of Memory 2 data revealed a main effect of condition, $F(1,10)=35.22, p<.001$, indicating that happy memories were rated by a coder 
as more positive than neutral memories $(d=1.98)$. Descriptive statistics are presented in Table 1.

Consistent with Aim 1 hypotheses, examination of the memory specificity ratings across the training, revealed a main effect of time, $F(2,20)=5.54, p=.01$, partial $\eta^{2}=.36$, but no main effect of condition or condition by time interaction ( $p s>.10$, partial $\left.\eta^{2}<.10\right)$. Across conditions, participants demonstrated an increase in specificity over the course of the training (see Table 1). By the end of the training, 100\% were able to recall specific happy or neutral memories. Memory valence did not differ as a function of memory specificity (all $p s>$.05). Likewise, the quality of participants' memories was not significantly associated the severity of their depression symptoms ( $p s>.05)$.

\section{Subjective Affect Following Memory Recall}

Subjective affect ratings are depicted in Figure 1. Examination of the impact of Memory 1 recall on subjective sadness revealed a main effect of condition, $F(1,25)=20.55$, $p<.001$, partial $\eta^{2}=.45$, and a main effect of time, $F(2,50)=4.46, p=.02$, partial $\eta^{2}=.15$, but no interaction $\left(p>.10\right.$, partial $\left.\eta^{2}=.02\right)$. As expected, PMET participants (who recalled sad memories) demonstrated more sadness than control participants (who recalled neutral memories). Additionally, across conditions, participants reported less sadness after Cue 4, than after Cue $8, t(26)=3.61, p<.001, d=.44$. When subjective happiness ratings were examined following the recall of Memory 1, results revealed a main effect of condition, $F(1$, $25)=16.49, p<.001$, partial $\eta^{2}=.40$. Across the training, when PMET participants recalled sad memories they reported significantly less happiness than participants in the control condition, who recalled neutral memories. No main effect of time (partial $\eta^{2}=.05$ ) or time by condition effects (partial $\left.\eta^{2}=.03\right)$ were found $(p s>.10)$ 
Results of the ANOVA assessing change in sadness from Memory 1 to Memory 2 produced a main effect of condition, $F(1,25)=14.46, p<.001$, partial $\eta^{2}=.37$, but no main effect of time (partial $\eta^{2}=.04$ ) or condition by time interaction (partial $\eta^{2}=.08$; both $p s>$ .10). Among PMET participants (who recalled positive memories for Memory 2), sadness ratings decreased from Memory 1 to Memory 2 at Cue $1, t(12)=2.41, p=.02, d=1.00$, Cue $4, t(12)=2.36, p=.02, d=1.00$, and Cue 8, $t(12)=4.29, p<.001, d=1.67$. In contrast, no differences in sadness ratings were seen following the neutral memories recalled for Memory 1 and Memory 2 among control participants ( $p s>.10, d s .13-.28$ ). Results also revealed a main effect of condition on happiness difference scores, $F(1,25)=16.77, p<$ .001 , partial $\eta^{2}=.40$, but no other significant effects (time: partial $\eta^{2}=.04$; time $\mathrm{x}$ condition: partial $\eta^{2}=.03 ; p s>.10$ ). PMET participants reported increases in happiness from Memory 1 to Memory 2 at Cue $1, t(12)=2.34, p=.02, d=1.12$, Cue $4, t(12)=2.62, p=.009, d=$ 1.13 , and Cue $8, t(12)=4.53, p<.001, d=1.85$. No differences were seen between Memory 1 and Memory 2 happiness ratings among control participants ( $p s>.10$, ds .01-.22).

Memory 2 affect ratings were subsequently examined as a function of condition at each of the three time points; consistent with hypothesis $2 \mathrm{~b}$, sadness and happiness ratings were not found to differ across PMET and control participants ( $p s>.10, d s$.03-.72). Evidence of carry-over effects from Memory 1 to Memory 2, as well as from Cue to Cue, was not observed.

\section{Perceived Ability to Relive Memories and Associations with Affect}

Examination of PMET participants' perceived ability to relive happy memories produced a marginally significant effect of time, $F(2,24)=2.96, p=.07$, partial $\eta^{2}=.04$. In line with hypothesis $3 a$, perceived ability to relive memories increased from Cue $1(M=$ 
3.92, $S E=.21)$ to Cue $4(M=4.38, S E=.24), t(12)=2.52, p=.01, d=.56$. However, perceived reliving did not increase from Cue 4 to Cue $8(M=4.38, S E=.26)$, t $(12)<.001, p$ $=1.00, d=0$. Of note, analyses examining a 2x8 ANOVA produced a statistically significant omnibus effect, $F(7,84)=2.13, p=.049$, partial $\eta^{2}=.01$. Probing of this effect revealed a cubic trend in the data, $F=7.61, p=.02$, with an increase from Cue 1 to Cue 4 , a decrease from Cue 4 to 6, and then an increase again for Cues 7 and 8.

Correlational analyses also provided partial support for hypothesis $3 \mathrm{~b}$. Perceived ability to relive the memory at Cue 1 was not significantly related to happiness ratings at any of the three time points (all $p s>.10$ ). However, perceived ability to relive the memory at Cue 4 was marginally related to greater subjective happiness ratings at that time point $(r$ $=.54, p=.06)$. Moreover, perceived ability to relive the memory at Cue 8 was associated with significantly greater subjective happiness at both Cue $4(r=.64, p=.02)$ and Cue $8(r=$ $.58, p=.04)$

\section{Discussion}

This study was a preliminary evaluation of PMET as a method for promoting mood regulation via the enhancement of positive memories among individuals with MDD. Results supported the first hypothesis that training procedures would improve the quality of memories across PMET and control conditions. Consistent with previous research (Gupta \& Kar, 2012; Williams et al., 2007; Young \& Erickson, 2012), participants in both conditions demonstrated initial difficulty recalling detailed and specific memories (only $50 \%$ of Cue 1 memories were specific). However, by the end of the training procedures, $100 \%$ of memories described a detailed event occurring on a particular day. Whereas the mechanism underlying improved specificity remains unknown, previous studies have 
found that repeated instruction helps individuals with depression recall vivid and specific memories (Yanes, Roberts, \& Carlos, 2008). From a clinical perspective, these results support the use of CBM over a single session of psychoeducation about skill use.

The second study aim was to examine how training influenced participants' subjective affective experience. Among participants in the PMET condition, recalling a sad autobiographical memory elicited greater sadness and less happiness, as compared to individuals in the control condition who were instructed to recall a neutral autobiographical memory. Results revealed that PMET participants were able to successfully repair induced negative mood states via the recall of positive memories, as evidenced by consistent decreases in sadness and increases in happiness from Memory 1 to Memory 2, as well as by the fact that subjective affect did not differ between conditions following Memory 2.

Interestingly, PMET participants' ability to repair the induced negative mood was evident from the very first cue. Given previous research (Joormann et al., 2007), we had expected that mood repair effects would require repeated training trials. One possible explanation for the current results is that PMET participants had already practiced recalling positive memories in a vivid and specific manner prior to the training phase. Clinically, results suggest that teaching depressed persons to effectively use positive memories to regulate negative emotions may be more feasible than was initially thought. Nonetheless, given the structure of the PMET procedures, the possibility that mood repair effects would have occurred spontaneously cannot be ruled out. The inclusion of a pretraining assessment of subjective affect following negative and positive memory recall could address this alternative hypothesis in the future. 
Conceptually, we had thought that PMET would influence subjective affect, in part, by overcoming participants' bias to recall memories in an overgeneral manner (Watkins et al., 2009; Williams et al., 2007). However, participants were able to repair their moods even at the beginning of the training, when positive memories were not often specific. Moreover, though positive memories became more specific throughout the training, participants' ability to repair their moods did not change. Given these results, considering other mechanisms that may be linked with effective mood repair seems wise. Beyond OGM, use of a field, rather than an observer perspective may increase participants' perceived ability to relive positive memories and allow them to more effectively utilize positive memory recall as an emotion regulation strategy (e.g., Holmes et al., 2008).

Results addressing this hypothesis showed that PMET participants demonstrated an increase in their ability to relive positive memories from the beginning to the midpoint of the training (though participants' ability decreased slightly for Cues 5 and 6, before increasing again for Cues 7 and 8). Further, associations between participants' ability and experienced positive affect emerged over the course of the training. There was also evidence of a recursive relationship between these constructs, such that happiness ratings at Cue 4 were associated with increased perceived ability to relive the memory at Cue 8 . Taken together, these results suggest that one's ability to relive a positive memory may be directly related to his or her ability to use positive memory recall as an emotion regulation skill. Future studies should consider how this construct may be more directly targeted by PMET procedures.

The current study was not without limitations. For example, the order of cues was standardized across participants and our results suggested that some cues generated 
memories with stronger emotional responses, or were associated with more difficulty reliving memories, than other cues did. Individuals in both conditions experienced significantly more sadness in response to memories generated at Cue 8 , as compared to memories generated at Cue 4. Likewise, participants appeared to have more difficulty reliving memories in response to Cues 5 and 6, as compared to Cues 4, 7, and 8 . Because evidence of mood repair emerged from the first cue, there is little reason to believe that cue order impacted study results. However, future studies should consider presenting cues in a counterbalanced or random order. Additionally, given the preliminary nature of the study, it only included subjective assessments of constructs of interest. Studies utilizing multimodal assessments with both subjective and objective (e.g., psychophysiological) measures would make a significant contribution to the literature.

The study was also limited by its small sample size and the fact that audio recordings were only collected from a subsample of participants. However, the sample was similar in size those previously reported (Gupta \& Kar, 2012; Lemogne et al., 2006; Young \& Erickson, 2012). As support was found for a majority of hypotheses, sample size did not prevent the detection of most training effects. Still, some analyses (e.g., interaction effects) were likely underpowered to detect significant effects and future PMET research should include larger samples. We should also note that two participants were withdrawn from the study due to an inability to comply with procedures. Both participants were assigned to the PMET condition, suggesting that recalling sad and happy memories in a vivid and detailed manner may be especially difficult for depressed persons. Though these participants did not appear to differ from the rest of the sample with regard to demographic characteristics or symptom severity, it may be important to investigate other 
individual differences that could facilitate or interfere with the ability to complete PMET procedures. Additionally, investigators should consider providing a rationale prior to PMET to increase participant "buy in" and understanding of how and why recalling positive memories may be helpful for mood regulation.

Lastly, the current study did not include a non-psychiatric comparison group. We are thus prevented from drawing conclusions about how PMET would function within a sample of non-depressed individuals. Yet, given previous research indicating that nondepressed individuals have no difficulty recalling positive memories (Williams et al., 2007), or using positive memories to influence their subjective emotional experience (Joormann \& Siemer, 2004), inclusion of non-psychiatric individuals was not relevant to the research question at hand.

Despite its limitations, this study provided preliminary evidence to suggest that positive autobiographical memory biases can be modified among individuals with MDD, and that positive memory recall can be used to effectively repair acute negative moods in this population. PMET yielded promising results, demonstrating that individuals trained to recall positive memories in a vivid and detailed manner were able to relive such memories more fully and that this ability was directly associated to participants' subjective experience of positive affect. Though these results lay essential groundwork, there is much more work to be done. For example, it would be interesting to examine whether memory specificity is related to perceived ability to relive the memory, and if so, whether the ability to relive positive memories is a mediator of the relationship between memory specificity and effective mood repair. In addition, the generalizability of these results to real-world situations in which individuals with depression spontaneously experience acute negative 
affect is an open empirical question. In the future, investigators should examine whether training effects are maintained over time, whether PMET procedures result in spontaneous use of positive memory recall as an emotion regulation strategy, and how spontaneous use of positive memory recall may be more or less effective than spontaneous use of other emotion regulation strategies (e.g., Egloff, Schmukle, Burns, \& Schwerdtfeger, 2006; Gruber, Harvey, \& Gross, 2012; Stange, Hamilton, Fresco, \& Alloy, 2017) for regulating subjective and physiological components of emotion among individuals with depression. . 


\section{References}

Beard, C. (2011). Cognitive bias modification for anxiety: Current evidence and future directions. Expert Reviews of Neurotherapy, 11, 299-311. doi: 10.1586/ern.10.194

Beck, A. T. (1964). Thinking and depression: Theory and therapy. Archives of General Psychiatry, 10(6), 561-571. doi: 10.1001/archpsyc.1964.01720240015003

Beck, A. T. (2005). The current state of cognitive therapy: A 40-year retrospective. Archives of General Psychiatry, 62(9), 953-959. doi: 10.1001/archpsyc.62.9.953

Beck, A. T., Steer, R. A., \& Brown, G. K. (1996). Manual for the Beck Depression Inventory-II. San Antonio, TX: Psychological Corporation.

Brewin, C. R., Gregory, J. D., Lipton, M., \& Burgess, N. (2010). Intrusive images in psychological disorders: Characteristics, neural mechanisms, and treatment implications. Psychological Review, 117(1), 210-232. doi: 10.1037/a0018113

Claúdio, V., Aurélio, J. G., \& Machado, P. P. P. (2012). Autobiographical memories in major depressive disorder. Clinical Psychology and Psychotherapy, 19, 375-389. doi: 10.1002/ccp.751

Collins, L. M., Schafer, J. L., \& Kam, C. (2001). A comparison of inclusive and restrictive strategies in modern missing data procedures. Psychological Methods, 6(4), 330-351. doi: $10.1037 / / 1082-989 X .6 .4 .330$

Egloff, B., Schmukle, S. C., Burns, L. R., \& Schwerdtfeger, A. (2006). Spontaneous emotion regulation during evaluated speaking tasks: Associations with negative affect, anxiety expression, memory, and physiological responding. Emotion, 6, 356-366. doi: $10.1037 / 1528-3542.6 .3 .356$ 
Everaert, J., Koster, E. H. W., \& Derakshan, N. (2012). The combined cognitive bias hypothesis in depression. Clinical Psychology Review, 32(5), 413-424. doi: 10.1016/j.cpr.2012.04.003

First, M. B., Spitzer, R. L., Gibbon, M., \& Williams, J. B. W. (2002). Structured Clinical Interview for DSM-IV-TR Axis I Disorders, Research Version, Patient Edition with Psychotic Screen (SCID-I/P W/ PSY SCREEN). New York: Biometrics Research, New York State Psychiatric Institute.

Gruber, J., Harvey, A. G., \& Gross, J. J. (2012). When trying is not enough: Emotion regulation and the effort-success gap in bipolar disorder. Emotion, 12, 997-1003. doi: $10.1037 / \mathrm{a} 0026822$

Gupta, R., \& Kar, B. R. (2012). Attention and memory biases as stable abnormalities among currently depressed and currently remitted individuals with unipolar depression. Frontiers in Psychiatry, 3, 1-7. doi: 10.3389/fpsyt.2012.00099

Hallion, L. S., \& Ruscio, A. M. (2011). A meta-analysis of the effect of cognitive bias modification on anxiety and depression. Psychological Bulletin, 137(6), 940-958. doi: $10.1037 / \mathrm{a} 0024355$

Holmes, E. A., Coughtrey, A. E., \& Connor A. (2008). Looking at or through rose-tinted glasses? Imagery perspective and positive mood. Emotion, 8(6), 875-879. doi: $10.1037 / \mathrm{a} 0013617$

Holmes, E. A., Mathews, A., \& Dalgleish, T. (2006). Positive interpretation training: Effects of mental imagery versus verbal training on positive mood. Behavior Therapy, 37(3): 237-247. doi: 10.1016/j.beth.2006.02.002 . 
Joormann, J., \& Arditte, K. A. (2014). Cognitive aspects of depression. In: Gotlib IH, Hammen CL (Eds.), Handbook of Depression, $3^{\text {rd }}$ Ed. New York: Guilford Press (pp. 259-276).

Joormann, J., \& D'Avanzato, C. (2010). Emotion regulation in depression: Examining the role of cognitive processes. Cognition and Emotion, 24(6), 913-939. doi: $10.1080 / 02699931003784939$

Joormann, J., \& Siemer, M. (2004). Memory accessibility, mood regulation, and dysphoria: Difficulties in repairing sad mood with happy memories? Journal of Abnormal Psychology, 113(2), 179-188. doi: 10.1037/0021-843X.113.2.179

Joormann, J., Siemer, M., \& Gotlib, I. H. (2007). Mood regulation in depression: Differential effects of distraction and recall of happy memories on sad mood. Journal of Abnormal Psychology, 116(3), 484-490. doi: 10.1037/0021-843X.116.3.484

Lemogne, C., Piolino, P., Friszer, S., Claret, A., Girault, N., Jouvent, R. ... Fossati, P. (2006). Episodic autobiographical memory in depression: Specificity, autonoetic consciousness, and self-perspective. Consciousness and Cognition, 15, 258-268. doi: 10.1016/j.concog.2005.07.005

Neshat-Doost, H. T., Dalgleish, T., Yule, W., Kalantari, M., Ahmadi, S. J., Dyregrov, A., \& Jobson, L. (2012). Enhancing autobiographical memory specificity through cognitive training: An intervention for depression translated from basic science. Clinical Psychological Science, 1(1), 84-92. doi: 10.1177/2167702612454613

Peeters, F., Wessel, I., Merckelbach, H., \& Boon-Vermeeren, M. (2002). Autobiographical memory specificity and the course of major depressive disorder. Comprehensive Psychiatry, 43(5), 344-350. doi: 10.1053/comp.2002.34635 
Raes, F., Hermans, D., Williams, J. M. G., Demyttenaere, K., Sabbe, B., Pieters, G., \& Eelen, P. (2005). Reduced specificity of autobiographical memory: A mediator between rumination and ineffective social problem-solving in major depression? Journal of Affective Disorders, 87(2), 331-335. doi: 10.1016/j.jad.2005.05.004

Schafer, J. L., Graham, J. W. (2002). Missing data: Our view of the state of the art. Psychological Methods, 7(2), 147-177. doi: 10.1037//1082-989X.7.2.147

Shear, M. K., Greeno, C., Kang, J., et al. Diagnosis of nonpsychotic patients in community clinics. American Journal of Psychiatry, 157(4), 581-857. doi: 10.1176/appi.ajp.157.4.581

Stange, J. P., Hamilton, J. L., Fresco, D. M., \& Alloy, L. B. (2017). Flexible parasympathetic responses to sadness facilitate spontaneous affect regulation. Psychophysiology, advance online publication. doi: 10.1111/psyp.12856

Sumner, J. A., Griffith, J. W., \& Mineka, S. (2010). Overgeneral autobiographical memory as a predictor of the course of depression: A meta-analysis. Behaviour Resarch and Therapy, 48, 614-625. doi: 10.1016/j.brat.2010.03.013

Watkins, E. R., Baeyens, C. B., Read, R. (2009). Concreteness training reduces dysphoria: Proof-of-principle for repeated cognitive bias modification in depression. Journal of Abnormal Psychology, 118(1), 55-64. doi: 10.1037/a0013642

Williams, J. M. G., Barnhofer, T., Crane, C., Hermans, D., Raes, F., Watkins, E., \& Dalgleish, T. (2007). Autobiographical memory specificity and emotions disorder. Psychological Bulletin, 133(1), 122-148. doi: 10.1037/0033-2909.133.1.122 
Yanes, P. K., Roberts, J. E., \& Carlos, E. L. (2008). Does overgeneral autobiographical memory result from poor memory for task instructions. Memory, 16, 669-677. doi: $10.1080 / 09658210802135351$

Young, K. D., Erickson, K., \& Drevets, W. C. (2012). Match between cue and memory valence during autobiographical memory recall in depression. Psychological Reports: Mental \& Physical Health, 111(1), 129-148. doi: 10.2466/09.02.15.PR0.111.4.129-148 
Table 1. Mean (standard deviation) memory valence ratings and the percentage of generic vs. specific memories across the PMST, as a function of study condition.

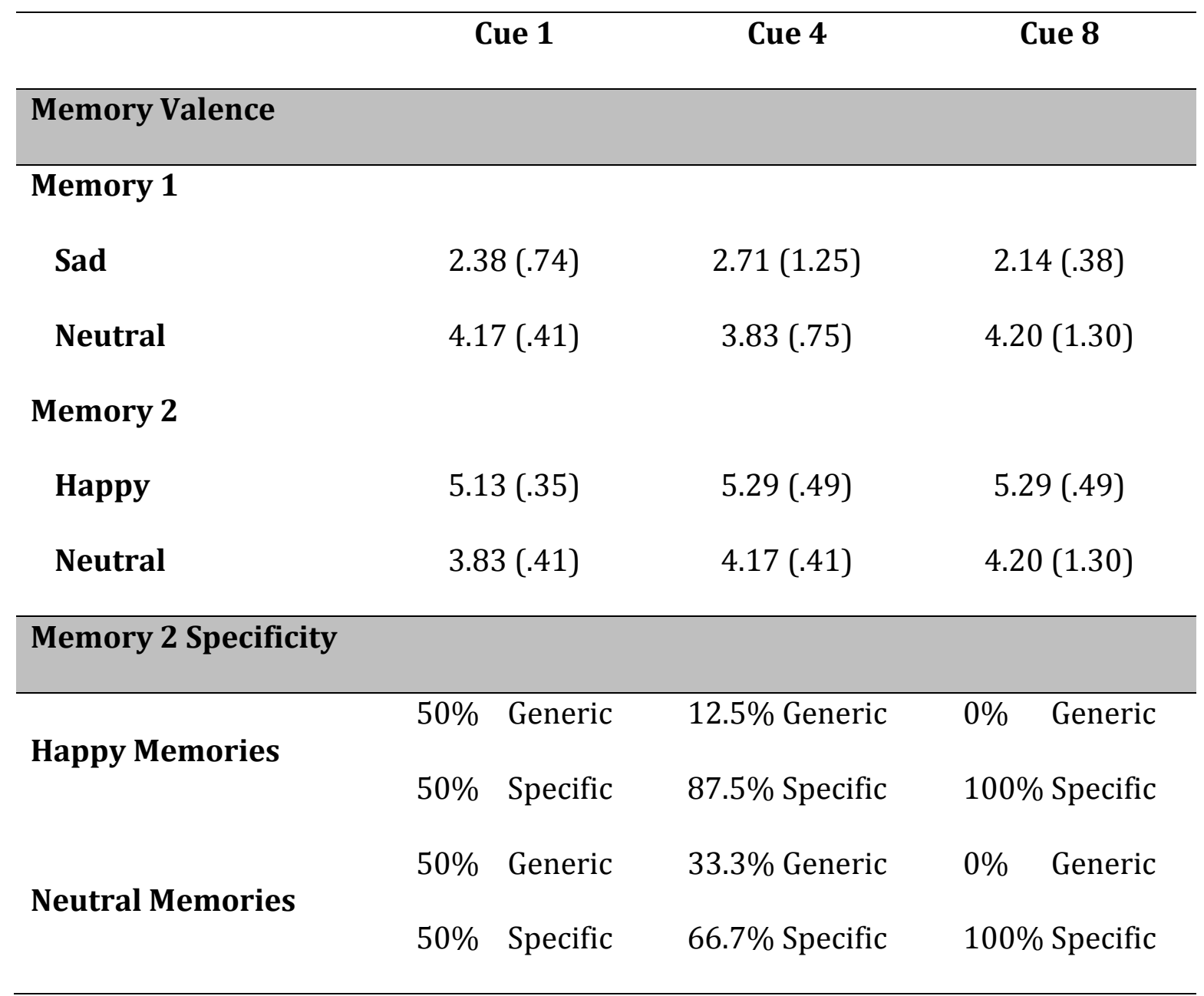

Note. Sad/happy memories refer to memories recalled by the experimental condition, whereas neutral memories refer to the corresponding memories recalled by the control condition. 


\section{Figure Captions}

Figure 1. Subjective sadness (Panel 1) and happiness (Panel 2) ratings as a function of memory and study condition. 